\title{
XPM-induced Crosstalk in SCM-WDM Passive Optical Networks
}

\author{
Aakash Kashyap \\ Department of ECE \\ MMEC, MMU, MULLANA, \\ AMBALA
}

\author{
Naresh Kumar \\ Department of ECE \\ NIT Hamirpur- India
}

\author{
Pooja Kaushik \\ Department of ECE \\ MMEC, MMU, MULLANA, \\ AMBALA
}

\begin{abstract}
In this paper, the XPM-induced crosstalk has been evaluated with third order Dispersion in SCM-WDM optical communication system at different optical powers for various parameters (dispersion, transmission length, effective area and modulation frequency) that play an important role in signal transmission. Results show that as the dispersion, transmission length and modulation frequency parameters increase, crosstalk increases with increase in optical powers and effective areas increase, crosstalk decreases with increase in optical power.
\end{abstract}

\section{General Terms}

XPM-induced Crosstalk, Dispersion, SCM-WDM.

\section{Keywords}

Sub-Carrier Multiplexing, Wavelength Division Multiplexing, Cross Phase modulation, Third- order dispersion, Transmission length, Crosstalk.

\section{INTRODUCTION}

Fiber-to-the-Home (FTTH) has been the main attraction in the telecommunication industry. Direct fiber connection has always been viewed as the long awaited solution due to the large bandwidth and low maintenance. However, in order for FTTH to remain competitive, a passive optical network is required. SCM is a potential solution for transmission in PONs [1]. The combination of SCM and WDM is a viable method to further increase the transmission capacity in PONs [2]. SCM-WDM systems, however suffer from non-linear effects in fiber. These non-linearities cause crosstalk between subscribers on different wavelengths. In a dispersive fiber, the dominant fiber nonlinearity that causes crosstalk is crossphase modulation (XPM). Fiber nonlinearities such as cross phase modulation (XPM) may generate significant amounts of nonlinear crosstalk between adjacent SCM channels because they are very closely spaced [3-4]. The effects of XPM and GVD in SCM-WDM video transmission systems while considering two WDM channels were reported [5]. Analytical investigation was analyzed in [6] with considering three WDM channels. The work reported in [7] considered the impact of second-order dispersion (2OD) only for SRS and XPM-induced crosstalk. With the advancement of communication systems, there is a trend of using higher modulation frequencies. So it is necessary to investigate the performance of optical transmission link at higher modulation frequencies including the higher-order dispersion coefficients. The work reported [8] considered the impact of 2OD and third-order dispersion (3OD) coefficients independently at different modulation frequencies. This paper extends the work by including various parameters (Dispersion, effective area, transmission length and modulation frequency) that play an important role in signal transmission in SCM-WDM

passive optical network at different optical powers. After describing the introduction in Section 1, the expression for XPM-induced crosstalk has been reported in Section 2. The results and discussion are mention in Section 3 and concluding remarks are given in section 4 .

\section{XPM -INDUCED CROSSTALK}

Here the modified analysis for XPM induced crosstalk has been reported by considering the third-order dispersion terms. The investigation is important for pulse of duration $\leq 0.1 \mathrm{ps}$ propagating over the fiber.

Consider two optical waves with identical polarization, copropagating in single mode fiber and take two coupled equation describing XPM under a slowly varying envelop are given by $[9,10]$

$$
\frac{\partial A_{1}}{\partial Z}+\frac{1}{V_{g_{1}}} \frac{\partial A_{1}}{\partial t}=\left(-j \gamma P_{2}-\frac{\alpha}{2}\right) A_{1}
$$

$$
\frac{\partial A_{2}}{\partial Z}+\frac{1}{V_{g_{2}}} \frac{\partial A_{2}}{\partial t}=\left(-j \gamma P_{2}-\frac{\alpha}{2}\right) A_{2}
$$

Where A1 $(z, t), i=1,2$ denote the slowly varying complex field envelop of each wave, $\gamma=$ nonlinearity coefficient. Therefore optical power at the input of fiber can be expressed as [11]

$$
P_{i}=P_{c}\left[1+m \cos \omega_{1} t\right]
$$

Where $\mathrm{i}=1\left(\lambda_{1}\right)$ or $2\left(\lambda_{2}\right)$ and $\lambda_{1}>\lambda_{2}, \mathrm{Pc}$ is the average optical power, $\mathrm{m}$ is the modulation index, cos $\omega \mathrm{t}$ is the modulation signal, $\omega$ is the angular frequency. Solving equation (1) and (2) of electric envelop by neglecting $\gamma$ for initial condition $z=$ 0 and $\mathrm{t}=\tau_{1}$

$$
A_{1}(z, t)=A_{1}\left(0, \tau_{1}\right) \exp \left(\frac{\alpha z}{2}\right)
$$


By substituting the result of $\mathrm{A} 1(\mathrm{z}, \mathrm{t})$ in the second coupled equation

$$
A_{2}\left(z, \tau_{2}\right)=A_{2}\left(0, \tau_{2}\right) \exp \left(-\frac{\alpha z}{2}\right) \exp \left(-2 j \gamma \int_{0}^{z} P_{1}\left(0, \tau_{2}+d_{12} z\right) e^{-\alpha z} d z\right)
$$

Where

$\psi=-2 \gamma \int_{0}^{z} P_{1}\left(0, \tau_{2}+d_{12} z\right) e^{-\alpha z} d z$

and $\tau_{1}=\tau_{2}+d_{12} z$

Then equation (5)

$$
A_{2}\left(z, \tau_{2}\right)=A_{2}\left(0, \tau_{2}\right) \exp \left(-\frac{\alpha z}{2}\right) \exp (-j \psi)
$$

Considering group velocity dispersion and third order dispersion can be converted into intensity modulation via relation [12-13]

$P_{2}\left(z, \tau_{2}\right)=P_{2}\left(0, \tau_{2}\right)_{2}\left|\left\{1+j\left(\frac{\partial^{2} \psi}{\partial t^{2}}+j\left(\frac{\partial \psi}{\partial t}\right)^{2}\right) F_{1}+1+\frac{\partial^{3} \psi}{\partial t^{3}}-3 \frac{\partial^{2} \psi}{\partial t^{2}} \frac{\partial \psi}{\partial t}-j\left(\frac{\partial \psi}{\partial t}\right)^{3}\right) F\right|^{2}$

Where $F_{1}=-\beta_{2} \frac{z}{2}, F_{2}=-\beta_{3} \frac{z}{6}$

$\beta_{2}=\frac{\partial^{2} \beta}{\partial \omega^{2}}, \beta_{3}=\frac{\partial^{3} \beta}{\partial \omega^{3}}$

$\beta$ is the phase constant at wavelength $\lambda$, solving equation (7) we obtain

$$
P_{2}\left(z, \tau_{2}\right)=P_{2}\left(0, \tau_{2}\right)\left\{\left(1-2 F_{1}-6 F_{2} \frac{\partial \psi}{\partial t}\right) \frac{\partial^{2} \psi}{\partial t^{2}}\right\}
$$

Since the value of $\beta_{2}^{2}$ and $\beta_{3}^{2}$ are very small, they are neglected

$$
P_{2}\left(z, \tau_{2}\right)=P_{2}\left(0, \tau_{2}\right)\left\{1-2 F_{1}-6 F_{2} \frac{\partial \psi}{\partial t}\right\} \frac{\partial^{2} \psi}{\partial t^{2}}
$$

$$
P_{2}\left(z, \tau_{2}\right)=P_{2}\left(z, \tau_{2}\right) e^{-\alpha z}\left[\beta_{2}+\beta_{3} \frac{\partial \psi}{\partial t}\right] \frac{\partial^{2} \psi}{\partial t^{2}}
$$

We define here the following dispersion parameters [11]

$$
\beta_{2}=\frac{\lambda_{2}}{2 \pi c} D
$$

is the second-order dispersion parameter .

$$
\beta_{3}=\frac{\lambda^{2}}{(2 \pi c)^{2}}\left[\lambda^{2} D_{1}+2 \lambda D\right]
$$

is the third-order dispersion parameter. From equation (11) the effect of $\beta_{3}$ in $\partial P_{2}\left(z, \tau_{2}\right) / \partial z$ is given by $\mathrm{V}$.

\section{Case-1 (XPM- induced crosstalk with 3OD)}

The XPM-induced crosstalk due to 3OD coefficient is given by

$$
V=-\beta_{3} P_{c} e^{-\alpha z}\left[\frac{\partial \psi}{\partial \tau_{2}} \frac{\partial^{2} \psi}{\partial \tau_{2}^{2}}\right]
$$

XPM-induced crosstalk due to $3 \mathrm{OD}$ at wavelength $\lambda_{2}$ is given by

$$
V=-\frac{2 m \beta_{3} \gamma^{2} P_{c} \omega^{3}}{\left(\alpha-j \omega d_{12}\right)^{3}}\left(3+2 \alpha L+4 e^{-L \alpha} e^{j \omega d_{12} L}-e^{-2 L \alpha} e^{j \omega d_{12} L}-2 j \omega d_{12} L\right)
$$

$$
\begin{aligned}
& V=-\frac{2 m \beta_{3} \gamma^{2} P_{c} \omega^{3}}{\left(\alpha-j \omega d_{12}\right)^{3}}\left\{\left(3+2 \alpha L+4 e^{-\alpha L} \cos \left(\omega d_{12} L\right)-e^{-2 \alpha L} \cos \right.\right. \\
& \left.\left.\left(2 \omega d_{12} L\right)+j 4 e^{-\alpha L} \sin \left(\omega d_{12} L\right)-e^{-2 \alpha L} \sin \left(2 \omega d_{12} L\right)-2 \omega d_{12} L\right)\right\}
\end{aligned}
$$

\section{RESULT ANALYSIS}

Here, the results have been mentioned for XPM-induced crosstalk at various optical powers in the presence of third order dispersion in SCM-WDM communication systems. The results have been reported by taking values of the various parameters like: $\Delta \lambda=4 \mathrm{~nm}, \mathrm{~L}=50 \mathrm{~km}, n_{2}=2.68 \mathrm{e}-20 \mathrm{~m}^{2} / \mathrm{W}$, $\gamma=2 \pi n_{2} / \lambda_{2} A_{\text {eff }}, \alpha=0.25 \mathrm{dBm}, \lambda_{1}=1542 \mathrm{~nm}$ and $\lambda_{2}=1546 \mathrm{~nm}$ and The values of $\mathrm{D}=17 \mathrm{ps} / \mathrm{nm} / \mathrm{km}, \mathrm{D}_{1}=0.085$ $\mathrm{ps} / \mathrm{nm} / \mathrm{km}$.

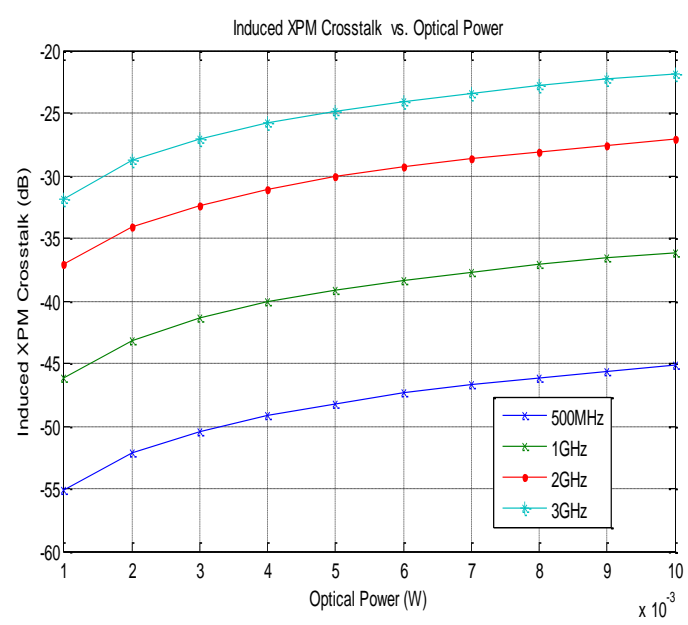

Fig.1. the graph between induced XPM Crosstalk versus Optical power at different modulation frequency.

Figure1 depicts the XPM-induced crosstalk versus optical power at varied modulation frequency. The XPM-induced crosstalk increases exponentially with the increase in optical power Fig. 1 shows that the XPM-induced crosstalk is (-55 to $45),(-46$ to -36$),(-37$ to -26$)$ and $(-32$ to -24$) \mathrm{dB}$ in the presence of modulation frequency $500 \mathrm{MHz}, 1 \mathrm{GHz}, 2 \mathrm{GHz}$ and $3 \mathrm{GHz}$ at $10 \mathrm{~mW}$ optical power. 


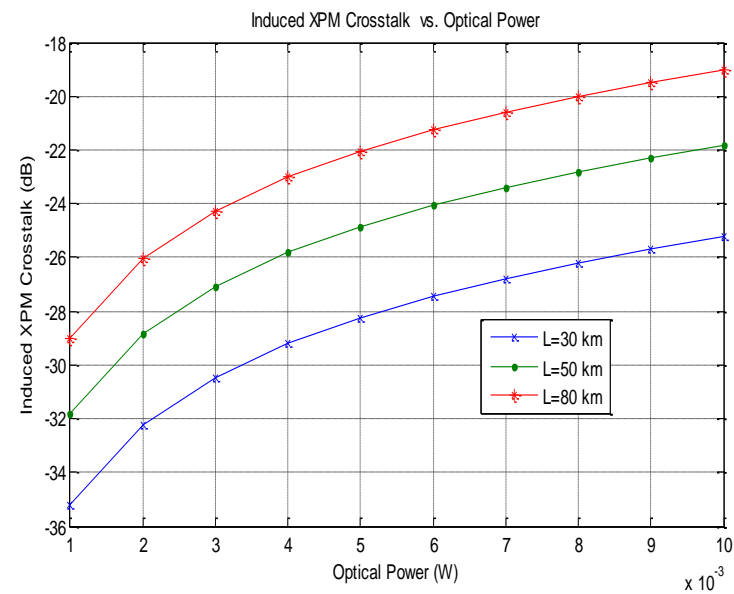

Fig.2. the graph between induced XPM Crosstalk versus optical power at different transmission length.

Furthermore Figure.2 illustrates the exponential growth in the XPM-induced crosstalk versus optical power at varied transmission length. The Fig.2 shows that the XPM-induced crosstalk is (-35 to -25$),(-32$ to -22$)$ and (-29 to -19$) \mathrm{dB}$ in the presence of transmission length 30,80 and $120 \mathrm{~km}$ at $10 \mathrm{~mW}$ optical power.

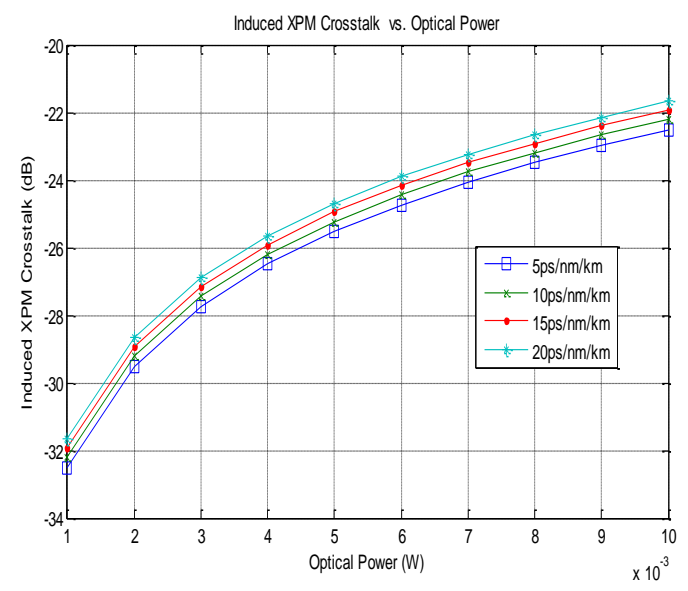

Fig.3. the graph between induced XPM Crosstalk versus optical power at different Dispersion parameter.

Furthermore Figure.3 illustrates the exponential growth in the XPM-induced crosstalk versus optical power at varied different dispersion parameter. The Fig.3 shows that the XPM-induced crosstalk is (-33 to -23$),(-32.5$ to -22.5$),(-32$ to $-22)$ and $(-31.8$ to -21.5$) \mathrm{dB}$ in the presence of dispersion parameter 5, 10, 15 and $20 \mathrm{ps} / \mathrm{nm} / \mathrm{km}$ at optical power.

Similarly Figure.4 illustrates the exponential growth in the XPM-induced crosstalk versus optical power at varied effective areas. The Fig.4 shows that the XPM-induced crosstalk is (-30 to -20.5$),(-32$ to -22$)$ and (-34 to -23$) \mathrm{dB}$ in the presence of effective area 55, 80 and 120 at optical power.

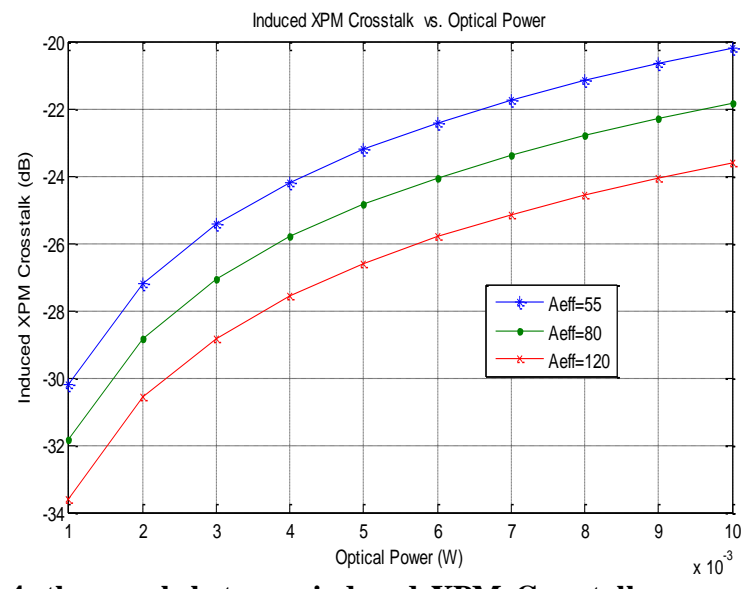

Fig.4. the graph between induced XPM Crosstalk versus optical power at different effective area.

\section{CONCLUSION}

This paper presents the detailed theoretical analysis the influence of third-order dispersion effect on XPM-induced crosstalk. It observed that the different parameter (Dispersion, effective area, transmission length and modulation frequency) has significant impact on XPM-induced crosstalk. At $6 \mathrm{~mW}$ optical power, the XPM-induced crosstalk is -25, -24.5, 24.1 and $-23.7 \mathrm{~dB}$ in the presence of dispersion $5,10,15,20$ $\mathrm{ps} / \mathrm{nm} / \mathrm{km}$, the XPM-induced crosstalk is $-27,-24$ and $-21 \mathrm{~dB}$ in the presence of transmission length 30,50 and $80 \mathrm{~km}$ at $6 \mathrm{~mW}$ optical power, the XPM-induced crosstalk is $-47,-38,-29$ and $-24 \mathrm{~dB}$ in the presence of modulation frequency $500 \mathrm{MHz}$, $1 \mathrm{GHz}, 2 \mathrm{GHz}$ and $3 \mathrm{GHz}$ at $6 \mathrm{~mW}$ optical power and the XPMinduced crosstalk is $-23,-24$ and $-25.6 \mathrm{~dB}$ in the presence of effective area 55, 80 and 120 at $6 \mathrm{~mW}$ optical power. Studies established that there is significant impact of Higher order Dispersion with overall performance of optical transmission link therefore it is recommended that selection of proper optical fiber parameter needs to be taken care of. It is therefore concluded that as the dispersion, transmission length and modulation frequency parameter increase, crosstalk increases with increase in optical powers and effective areas increase, crosstalk deceases with increase in optical power.

\section{REFERENCES}

[1] Gros, R. and Olshansky, R. 1990. Multichannel coherent FSK experiments using subcarrier multiplexing techniques, IEEE Journal of Light wave Technology, 8 (1990) 406-415.

[2] Hui, R., Zhu, B., Huang, R., Allen, C., Demarest, K. and Roberts, D. 2001. 10Gb/s SCM systems using optical single side band modulation. Paper MM4, OFC'2001, Anaheim, CA, March 2001.

[3] Hui, R., Demarest, K., Allen, C. 1999. Cross phase modulation in multi-span WDM optical fiber systems, IEEE Journal of Light wave Technology, Vol. 17 (7) (1999) 1018-1026.

[4] Ho, K. P. and Kahn, J. M. 1996. Method for cross talk measurement and reduction in Dense WDM systems, Journal of Light wave Technology, Vol. 14 (June 1996) 1127-1135.

[5] Wang, Z., Bodtker, E. and Jacobsen, G., Effects of Cross Phase Modulation in Wavelength Multiplexed SCM video transmission systems, Electronic Letters, 31 (1995) $1591-1592$ 
[6] Subramaniam, S., Abbou, F. M., Chuah, H. T. and Dambul, K.D., Performance evaluation of SCM-WDM microcellular communication system in the presence of XPM, IEICE Electronic Express, 2 (2005) 192-197.

[7] Frank S. Yang, Michel E. Marhic, Leonid G. Kazovsky, 2000. Nonlinear crosstalk and two countermeasures in SCM-WDM Optical communication systems, Journal of Light wave Technology Vol. 18 (4) (2000) 512-520.

[8] Arya, S. K., Sharma, A. K., Agarwala, R. A. 2009. Impact of 2OD and 3OD on SRS- and XPM-induced crosstalk in SCM-WDM optical transmission link, Optik, 120 (2009) 364-369.

[9] Agarwal, G. P. Application of Nonlinear Fiber Optics, Academic Press, San Deigo, CA, 2001.
[10] Peterman, K. 1990. FM-AM noise conversion in dispersive single mode fiber transmission lines, Electron. Letter Vol.26 (1990) 2097-2098.

[11] Sharma, A. K., Sinha, R. K., Agarwal, R. A. 1997. Improved analysis of dispersion compensation using differential time delay for high-speed long-span optical link, Fiber Integr. Opt., USA Vol.16 (1997) 415-426.

[12] Sharma, A. K., Sinha, R. K., Agarwala, R. A. 1998. Higher- order dispersion compensation by differential time delay, Optical Fiber Technology, USA Vol. 4 (1998)135-143.

[13] Singh, A., Sharma, A. K., Kamal, T. S. 2008. Four wave mixing analysis in WDM optical communication systems with higher-order dispersion, Optik 119, (2008) 788 792. 